Journal of Mathematics and Statistics 8 (2): 216-220, 2012

ISSN 1549-3644

C 2012 Science Publications

\title{
Mixed Formulation for a Signorini Problem
}

\author{
${ }^{1}$ Tedjani Hadj Ammar and ${ }^{2}$ Benabderrahmane Benyattou \\ ${ }^{1}$ Department of Mathematics and Informatics, \\ Faculty of Sciences Technology and Material Sciences, \\ Ouargla University, Ouargla (30000), Algeria \\ ${ }^{2}$ Department of Mathematics and Computer Sciences, Faculty of Sciences, \\ Laboratory of Computer Science and Mathematics, \\ Laghouat University, Laghouat (03000), Alegria
}

\begin{abstract}
Problem statement: This study deals with the study of a Signorini Problem (SP) of unilateral contact between two a linear or non linear elastic bodies. Approach: We present two variational formulations noted $\mathrm{P}_{1}, \mathrm{P}_{2}$, of the considered problem, where $\mathrm{P}_{1}$ depends on the displacement field and $\mathrm{P}_{2}$ depends on the stress field. In the linear case, under assumptions, then the considered problem was equivalent to a mixed variational formulation problem, where the unknowns are the displacement field and the normal constraint stress on the contact area. Results: Problems $\mathrm{P}_{1}$ and $\mathrm{P}_{2}$ were formally equivalent to the Signorini Problem (SP) and using Lions-Stampachia Theorem, we shown the existence and uniqueness result. Conclusion: The Signorini problem (PS) has a unique variational solution.
\end{abstract}

Key words: Signorini conditions, unilateral contact, mixed formulation, variational inequality

\section{INTRODUCTION}

The modeling of contact problems between two deformable bodies depends mainly the mechanical properties of materials considered as boundary conditions of contact. Among the different types of problems considered include problems of bilateral or unilateral contact without friction for elastic body. The accumulation of experimental data shows the limitations of the classical laws of friction both in terms of mathematical mechanics. Variational formulations and results of existence and uniqueness have been obtained in (Drabla, 1999) and in (Djabi et al., 1998) in the case of a frictionless contact problem between an elastic body and a rigid foundation and (Hild and Laborde, 2002; Amassad and Sofonea, 1998), for a frictionless contact problem between two deformable bodies using the quadratic finite elements method. Our aim in this study is to study the existence and uniqueness of a variational solution for a frictionless contact between two elastic bodies based on a mixed formulation, this problem known as problem of Signiorin.

Problem statement: Let us consider two elastic bodies, occupying two bounded domains $\Omega^{1}, \Omega^{2}$ of $\mathrm{IR}^{\mathrm{N}}, \mathrm{N}=2$,

3. The boundary $\Gamma^{\ell}=\partial \Omega^{\ell}$ is assumed piecewise continuous and composed of three complementary parts $\Gamma_{1}^{\ell}, \Gamma_{2}^{\ell}$ and $\Gamma_{3}^{\ell}$. The body $\bar{\Omega}^{\ell}$ is fixed on the set $\Gamma_{1}^{\ell}$ of positive measure. The $\Gamma_{2}^{\ell}$ boundary is submitted to a density of forces noted $\mathrm{g}^{\ell}$ and the body forces are denoted by $\mathrm{f}^{\ell}$. In the initial configuration, both bodies have a common contact portion $\Gamma_{3}^{1}=\Gamma_{3}^{2}=\Gamma_{3}$. The normal unit outward vector on $\Omega^{1}$ is denoted $\eta^{1}$. Mathematical relations in a mechanical problem can be divided into two kinds: one of them consists of materialindependent relations and the other material-dependent relations, or constitutive laws, the material-independent relations include the strain-displacement relation, the equation of equilibrium and boundary conditions. The equation of equilibrium takes the form Eq. 1:

$\operatorname{div} \sigma^{\ell}+\mathrm{f}^{\ell}=0$ in $\Omega^{\ell}$

where, $\sigma^{\ell}$ represents the stress tensor field. The specified boundary conditions take the form Eq. 2 and 3:

$$
\mathrm{u}^{\ell}=0 \text { on } \Gamma_{1}^{\ell}
$$

$$
\sigma^{\ell} \eta^{\ell}=\mathrm{g}^{\ell} \text { on } \Gamma_{2}^{\ell}
$$

Corresponding Author: Tedjani Hadj Ammar, Department of Mathematics and Informatics,

Faculty of Sciences Technology and Material Sciences, Ouargla University, Ouargla (30000), Algeria 
The elastic constitutive law of the material is assumed to be Eq. 4:

$\sigma^{\ell}=\mathrm{F}^{\ell}\left(\varepsilon\left(\mathrm{u}^{\ell}\right)\right)$ in $\Omega^{\ell}$

In which $\mathrm{F}^{\ell}$ is the given linear or nonlinear function. The conditions on the boundary part $\Gamma_{3}$ constrained by frictionless unilateral contact conditions incorporate the Signorini conditions Eq. 5:

$$
\begin{cases}\text { (a) } & \sigma_{\eta}^{1}=\sigma_{\eta}^{2} \equiv \sigma_{\eta} \\ \text { (b) }\left[u_{\eta}\right] \leq 0, \sigma_{\eta} \leq 0, \quad \sigma_{\eta}\left[u_{\eta}\right]=0 \quad \text { on } \Gamma_{3} \\ \text { (c) } \sigma_{\tau}^{1}=\sigma_{\tau}^{2}=0\end{cases}
$$

In the study of the problem (1)-(5), we assume that the elasticity operator:

$$
\mathrm{F}^{\ell}: \mathrm{S}_{\mathrm{N}} \rightarrow \mathrm{S}_{\mathrm{N}}
$$

Satisfies Eq. 6-8:

$$
\begin{aligned}
& \left\{\begin{array}{l}
\text { (a) } \quad \exists \mathrm{m}>0: \quad\left(\mathrm{F}^{\ell}\left(\varepsilon_{1}\right)-\mathrm{F}^{\ell}\left(\varepsilon_{2}\right)\right)\left(\varepsilon_{1}-\varepsilon_{2}\right) \geq \mathrm{m}\left|\varepsilon_{1}-\varepsilon_{2}\right|^{2} \\
\text { (b) } \exists \mathrm{L}>0: \quad\left|\mathrm{F}^{\ell}\left(\varepsilon_{1}\right)-\mathrm{F}^{\ell}\left(\varepsilon_{2}\right)\right| \leq \mathrm{L}\left|\varepsilon_{1}-\varepsilon_{2}\right| \\
\text { (c) } \quad \mathrm{F}^{\ell}(0)=0
\end{array}\right. \\
& \mathrm{f}^{\ell} \in \mathrm{IL}^{2}\left(\Omega^{\ell}\right)^{\mathrm{N}} \\
& \mathrm{g}^{\ell} \in \mathrm{IL}^{2}\left(\Gamma_{2}^{\ell}\right)^{\mathrm{N}}
\end{aligned}
$$

Using the notation $\mathcal{H}^{\ell}=\mathbb{L}^{2}\left(\Omega^{\ell}\right)^{\mathrm{N} \times \mathrm{N}}$ and from (6) we obtain that for all $\tau^{\ell} \in \mathcal{H}^{\ell}$, with the function $\mathrm{x}^{\ell} \mapsto \mathrm{F}^{\ell}\left(\mathrm{x}^{\ell}\right)$ belongs to $\mathcal{H}^{\ell}$ and hence we may consider $\mathrm{F}^{\ell}$ as an operator defined on $\mathcal{H}^{\ell}$ with the range on $\mathcal{H}^{\ell}$. Moreover, $\mathrm{F}: \mathcal{H}^{\ell} \rightarrow \mathcal{H}^{\ell}$ is a strictly monotone, Lipschitz and continuous operator. Therefore $\mathrm{F}^{\ell}$ is invertible and his inverse $\left(\mathrm{F}^{\ell}\right)^{-1}$ is also, a strictly monotone, Lipschitz and continuous operator.

Variational formulations: Let us introduce the following spaces Eq. 9:

$$
\left\{\begin{array}{l}
\mathrm{V}\left(\Omega^{\ell}\right)=\left\{\mathrm{v}^{\ell} \in \mathrm{H}^{1}\left(\Omega^{\ell}\right)^{\mathrm{N}} ; \mathrm{v}^{\ell}=0 \text { on } \Gamma_{1}^{\ell}\right\}, \ell=1,2 \\
\mathrm{~V}=\mathrm{V}\left(\Omega^{1}\right) \times \mathrm{V}\left(\Omega^{2}\right)
\end{array}\right.
$$

Since meas $\left(\Gamma_{1}^{\ell}\right)>0$, from Korn's inequality it follows Eq. 10:

$$
\left\|\varepsilon\left(v^{\ell}\right)\right\|_{\mathcal{H}^{\ell}} \geq \mathrm{m}^{\ell}\left\|v^{\ell}\right\|_{\mathrm{H}^{\ell}\left(\Omega^{\ell}\right)}, \quad \forall \mathrm{v}^{\ell} \in \mathrm{V}\left(\Omega^{\ell}\right)
$$

Here and below $\mathrm{m}^{\ell}$ denotes a strictly positive generic constant which may depend on $\Omega^{\ell}, \Gamma_{1}^{\ell}, \mathrm{F}^{\ell}$.

On $\mathrm{V}$ we consider the inner product given by Eq. 11:

$$
\langle v, w\rangle=\left\langle\varepsilon\left(v^{1}\right), \varepsilon\left(w^{1}\right)\right\rangle_{\mathscr{H}^{\dagger}}+\left\langle\varepsilon\left(v^{2}\right), \varepsilon\left(w^{2}\right)\right\rangle_{\mathscr{H}^{2}}
$$

and let \|\|$_{V}$ be the associated norm. It follows from (10) that \|\|$_{H_{1}}$ and \|\|$_{\mathrm{V}}$ are equivalent norms.

Therefore $\mathrm{V}$ is a real Hilbert space. Thus applying the Riesz representation theorem to claim that there exists a unique member $\varphi=\left(\varphi^{1}, \varphi^{2}\right) \in \mathrm{V}$ such that Eq. 12:

$$
\left\langle\phi^{\ell}, \mathrm{v}^{\ell}\right\rangle_{\mathrm{v}\left(\Omega^{\ell}\right)}=\int_{\Omega^{\ell}} \mathrm{f}^{\ell} \cdot \mathrm{v}^{\ell} \mathrm{d} \Omega^{\ell}+\int_{\Gamma_{2}^{\prime}} \mathrm{g}^{\ell} \cdot \mathrm{v}^{\ell} \eta^{\ell} \mathrm{d} \Gamma_{2}^{\ell}, \forall \mathrm{v}^{\ell} \in \mathrm{V}\left(\Omega^{\ell}\right) .
$$

Finally, we denote in the sequel by $\mathrm{U}_{\mathrm{ad}}$ the set of admissible displacement fields defined by Eq. 13:

$$
\mathrm{U}_{\mathrm{ad}}=\left\{\mathrm{v}=\left(\mathrm{v}^{1}, \mathrm{v}^{2}\right) \in \mathrm{V},\left.\quad\left[\mathrm{v}_{\mathrm{n}}\right]\right|_{\Gamma_{3}} \leq 0\right\} .
$$

Also, let $\sum_{\text {ad }}$ denotes the set of admissible stress fields given by Eq.14:

$\Sigma_{\text {ad }}=\left\{\begin{array}{l}\tau=\left(\tau_{1}, \tau_{2}\right) \in \mathcal{H} ; \\ \sum_{\ell=1}^{2}\left\langle\tau^{\ell}, \varepsilon\left(\mathrm{v}^{\ell}\right)\right\rangle_{\mathcal{H}^{\ell}} \geq\langle\phi, \mathrm{v}\rangle, \forall \mathrm{v} \in \mathrm{U}_{\mathrm{ad}}\end{array}\right\}$

Using (1)-(5) we have the following result.

Lemma 1: If $(u, \sigma)$ are sufficiently regular functions satisfying (1)-(5), then Eq. 15-17:

$$
\begin{aligned}
& \mathrm{u} \in \mathrm{U}_{\mathrm{ad}}, \sigma \in \Sigma_{\mathrm{ad}}, \\
& \sum_{\ell=1}^{2}\left\langle\sigma^{\ell}, \varepsilon\left(\mathrm{u}^{\ell}\right)-\varepsilon\left(\mathrm{u}^{\ell}\right)\right\rangle_{\mathrm{H}^{\ell}} \geq\langle\phi, \mathrm{v}-\mathrm{u}\rangle, \forall \mathrm{v} \in \mathrm{U}_{\mathrm{ad}},
\end{aligned}
$$

$$
\sum_{\ell=1}^{2}\left\langle\tau^{\ell}-\sigma^{\ell}, \varepsilon\left(\mathbf{u}^{\ell}\right)\right\rangle_{\mathrm{H}^{\ell}} \geq 0, \forall \tau \in \Sigma_{\text {ad }}
$$

Proof of lemma 1: The regularity $u \in U_{a d}$ follows from (2) and (5). Using Green's formula in (1), (2), (3), (12), we have (16).

Choosing now $v=2 u$ and $v=0$ in (16), we deduce Eq. 18: 


$$
\sum_{\ell=1}^{2}\left\langle\sigma^{\ell}, \varepsilon\left(\mathbf{u}^{\ell}\right)\right\rangle_{\mathrm{H}^{\ell}}=\langle\varphi, \mathrm{u}\rangle
$$

Fom (16), it finds:

$$
\sum_{\ell=1}^{2}\left\langle\sigma^{\ell}, \varepsilon\left(\mathrm{v}^{\ell}\right)\right\rangle_{\mathrm{H}^{\ell}} \geq\langle\varphi, \mathrm{v}\rangle
$$

Thus using (14) we deduce that $\sigma \in \sum_{\text {ad }}$. Using now (14) and (18) we find (17).

The Lemmal and (4) permit us to consider the following two variational problems.

Problem $\mathbf{P}_{1}$ : Find a displacement fields $u=\left(\mathrm{u}^{1}, \mathrm{u}^{2}\right)$, where $\mathrm{u}^{\ell}: \Omega^{\ell} \rightarrow \mathbb{R}^{\mathrm{N}}$ such that Eq. 19:

$$
\left\{\begin{array}{l}
\mathrm{u} \in \mathrm{U}_{\mathrm{ad}}, \\
\sum_{\ell=1}^{2}\left\langle\mathrm{~F}^{\ell}\left(\varepsilon\left(\mathrm{u}^{\ell}\right)\right), \varepsilon\left(\mathrm{v}^{\ell}\right)-\varepsilon\left(\mathrm{u}^{\ell}\right)\right\rangle_{\mathcal{H}^{\ell}} \geq\langle\phi, \mathrm{v}-\mathrm{u}\rangle, \forall \mathrm{v} \in \mathrm{U}_{\mathrm{ad}} .
\end{array}\right.
$$

Problem $\mathbf{P}_{2}$ : Find a stress fields $\sigma=\left(\sigma^{1}, \sigma^{2}\right)$, where $\sigma^{\ell}: \Omega^{\ell} \rightarrow \mathbb{S}_{\mathrm{N}}$ such that Eq. 20:

$$
\left\{\begin{array}{l}
\sigma \in \Sigma_{\text {ad }}, \\
\sum_{\ell=1}^{2}\left\langle\tau^{\ell}-\sigma^{\ell},\left(\mathrm{F}^{\ell}\right)^{-1}\left(\sigma^{\ell}\right)\right\rangle_{\mathcal{H}^{\ell}} \geq 0, \forall \tau^{\ell} \in \Sigma_{\text {ad }} .
\end{array}\right.
$$

We note that problems $\mathrm{P}_{1}$ and $\mathrm{P}_{2}$ are formally equivalent to the mechanical problem (1)-(5).

Let $\mathrm{u}=\left(\mathrm{u}^{1}, \mathrm{u}^{2}\right)$ be a solution of the variational problem $\mathrm{P}_{1}$ and $\sigma=\left(\sigma^{1}, \sigma^{2}\right)$ is defined by (4), then, using the arguments of (Hild and Laborde, 2002), it follows that $(u, \sigma)$ is a solution of the variational problem (1)-(5). Similarly, let $\sigma=\left(\sigma^{1}, \sigma^{2}\right)$ be a solution of the variational problem $\mathrm{P}_{2}$ and $\mathrm{u}=\left(\mathrm{u}^{1}, \mathrm{u}^{2}\right)$ is given by $\sigma^{\ell}=\mathrm{F}^{\ell}\left(\varepsilon\left(\mathrm{u}^{\ell}\right)\right)$, then $(\mathrm{u}, \sigma)$ is a solution of the variational problem (1)-(5).

Mixed variational formulation: In the linear case, we establish a new variational formulation of the problem (1)-(5), where the unknowns are the displacements field: $\mathrm{u}=\left(\mathrm{u}^{1}, \mathrm{u}^{2}\right)$ and the function $\lambda$ which is the normal component of the stress tensor field on $\Gamma_{3}$. The linear elastic constitutive law is given by Hooke's law for homogeneous and isotropy materials:

$$
\sigma^{\ell}=\mathrm{A}^{\ell} \varepsilon\left(\mathrm{u}^{\ell}\right)=\tilde{\lambda}^{\ell} \operatorname{tr}\left(\varepsilon\left(\mathrm{u}^{\ell}\right)\right) \mathrm{id}+2 \tilde{\mu} \varepsilon\left(\mathrm{u}^{\ell}\right)
$$

where, $\quad \mathrm{A}=\left(\mathrm{A}_{\mathrm{jipq}}^{\ell}\right), \ell=1,2$ denotes the fourth-order isotropy material for linear elasticity satisfying the usual symmetry and ellipticity conditions in elasticity:

$$
\left\{\begin{array}{l}
\mathrm{A}_{\mathrm{ijpq}}^{\ell}=\mathrm{A}_{\mathrm{jiqp}}^{\ell}=\mathrm{A}_{\mathrm{pqij}}^{\ell}, \\
\mathrm{A}_{\mathrm{ijpq}}^{\ell} \varepsilon_{\mathrm{ij}} \varepsilon_{\mathrm{pq}} \geq \mathrm{m}\left|\varepsilon_{\mathrm{ij}}\right|^{2}, \forall \varepsilon \in \mathrm{IR}^{\mathrm{N} \times \mathrm{N}} .
\end{array}\right.
$$

The set of admissible normal stresses on $\Gamma_{3}$ can be defined as:

$$
\mathrm{M}=\left\{\begin{array}{l}
\mu \in \mathrm{H}^{-\frac{1}{2}}\left(\Gamma_{3}\right) ;\langle\mu, \psi\rangle_{-\frac{1}{2}, \frac{1}{2}, \Gamma_{3}} \geq 0, \\
\forall \psi \in \mathrm{H}^{\frac{1}{2}}\left(\Gamma_{3}\right), \text { and } \psi \geq 0 \text { p.p on } \Gamma_{3}
\end{array}\right\}
$$

For any $\mathrm{u}, \mathrm{v} \in \mathrm{V}$ and for any $\mu \in \mathrm{M}$, we define:

$$
\left\{\begin{array}{l}
\mathrm{a}(\mathrm{u}, \mathrm{v})=\sum_{\ell=1}^{2} \int_{\Omega^{\ell}}\left\langle\mathrm{A}^{\ell} \varepsilon\left(\mathrm{u}^{\ell}\right), \varepsilon\left(\mathrm{v}^{\ell}\right)\right\rangle_{\mathcal{H}^{\ell}} \mathrm{d} \Omega^{\ell}, \\
\mathrm{b}(\mathrm{u}, \mu)=\int_{\Gamma_{3}} \mu\left(\mathrm{u}^{1} \cdot \eta^{1}+\mathrm{u}^{2} \cdot \eta^{2}\right) \mathrm{d} \Gamma_{3}, \\
\mathrm{~L}(\mathrm{v})=\sum_{\ell=1}^{2}\left(\int_{\Omega^{\ell}} \mathrm{f}^{\ell} \cdot \mathrm{v}^{\ell} \mathrm{d} \Omega^{\ell}+\int_{\Gamma_{2}^{\ell}} \mathrm{g}^{\ell} \cdot \mathrm{v}^{\ell} \eta^{\ell} \mathrm{d} \Gamma^{\ell}\right) .
\end{array}\right.
$$

Using (18) and Green's formula, we have the following result:

Lemma 2: For $\sigma^{\ell}=\mathrm{A}^{\ell} \varepsilon\left(\mathrm{u}^{\ell}\right), \ell=1,2$, if $\mathrm{u}=\left(\mathrm{u}^{1}, \mathrm{u}^{2}\right)$ is a solution to the problem (1)-(5), then Eq. 21-24:

$$
\begin{aligned}
& \sigma_{\eta}^{1}=\sigma_{\eta}^{2}(\equiv-\lambda) \text { on } \Gamma_{3} \\
& \lambda \in \mathrm{M} \\
& \mathrm{a}(\mathrm{u}, \mathrm{v})+\mathrm{b}(\mathrm{v}, \lambda)=\mathrm{L}(\mathrm{v}), \quad \forall \mathrm{v} \in \mathrm{V} \\
& \mathrm{b}(\mathrm{v}, \mu-\lambda) \leq 0, \quad \forall \mu \in \mathrm{M}
\end{aligned}
$$

Proof of lemma 2: The appurtenance of u to $\mathrm{H}_{1}$ allows easily deducing that $\sigma_{\eta} \in \mathrm{H}^{-\frac{1}{2}}\left(\Gamma_{3}\right)$. On another hand, taking into consideration the fact that $\sigma_{\eta} \leq 0$ on $\Gamma_{3}$, we deduce that $\lambda \geq 0$ on $\Gamma_{3}$, where $\lambda \in \mathrm{M}$.

Since $\sigma^{\ell}=\mathrm{A}^{\ell} \varepsilon\left(\mathrm{u}^{\ell}\right), \ell=1,2$, we have:

$$
\begin{aligned}
& \mathrm{a}(\mathrm{u}, \mathrm{v})+\mathrm{b}\left(\mathrm{v},-\sigma_{\eta}\right)=\int_{\Omega^{1}}\left\langle\sigma^{1}, \varepsilon\left(\mathrm{v}^{1}\right)\right\rangle \mathrm{d} \Omega^{1} \\
& +\int_{\Omega^{2}}\left\langle\sigma^{2}, \varepsilon\left(\mathrm{v}^{2}\right)\right\rangle \mathrm{d} \Omega^{2}-\int_{\Gamma_{3}} \sigma_{\eta}[\mathrm{v} \cdot \eta] \mathrm{d} \Gamma_{3}
\end{aligned}
$$


Using Green's formula, we obtain:

$$
\begin{aligned}
& \mathrm{a}(\mathrm{u}, \mathrm{v})+\mathrm{b}\left(\mathrm{v},-\sigma_{\eta}\right)= \\
& \sum_{\ell=1}^{2} \int_{\Omega^{\ell}} \mathrm{f}^{\ell} \mathrm{v}^{\ell} \mathrm{d} \Omega^{\ell}+\sum_{\ell=1}^{2}\left\langle\sigma^{\ell} \eta^{\ell}, \mathrm{v}^{\ell} \eta^{\ell}\right\rangle_{\mathrm{H}^{\frac{1}{2}}\left(\Gamma^{\ell}\right) \times \mathrm{H}^{\frac{1}{2}}\left(\Gamma^{\ell}\right)} \\
& -\int_{\Gamma_{3}} \sigma_{\eta}[\mathrm{v} \cdot \eta] \mathrm{d} \Gamma_{3}
\end{aligned}
$$

Remarking that $\sigma_{\eta}[\mathrm{v} . \eta]=0$ on $\Gamma_{3}$ and $\mathrm{v}^{\ell}=0$ on $\Gamma_{1}^{\ell}$, we have:

$$
\begin{aligned}
& \mathrm{a}(\mathrm{u}, \mathrm{v})+\mathrm{b}\left(\mathrm{v},-\sigma_{\eta}\right)=\int_{\Omega^{1}} \mathrm{f}^{1} \mathrm{v}^{1} \mathrm{~d} \Omega^{1}+\int_{\Gamma_{2}^{1}} \sigma_{\eta}^{1}\left(\mathrm{v}^{1} \cdot \eta^{1}\right) \mathrm{d} \Gamma_{2}^{1} \\
& +\int_{\Omega^{2}} \mathrm{f}^{2} \mathrm{v}^{2} \mathrm{~d} \Omega^{2}+\int_{\Gamma_{2}^{1}} \sigma_{\eta}^{2}\left(\mathrm{v}^{2} \cdot \eta^{2}\right) \mathrm{d} \Gamma_{2}^{2}
\end{aligned}
$$

Using $\sigma^{\ell} \eta^{\ell}=\mathrm{g}^{\ell}$ on $\Gamma_{2}^{\ell} ; \ell=1,2$, we find:

$$
\begin{aligned}
& \mathrm{a}(\mathrm{u}, \mathrm{v})+\mathrm{b}\left(\mathrm{v},-\sigma_{\eta}\right)=\int_{\Omega^{1}} \mathrm{f}^{1} \mathrm{v}^{1} \mathrm{~d} \Omega^{1}+\int_{\Gamma_{2}^{1}} \mathrm{~g}^{1}\left(\mathrm{v}^{1} \cdot \eta^{1}\right) \mathrm{d} \Gamma_{2}^{1} \\
& +\int_{\Omega^{2}} \mathrm{f}^{2} \mathrm{v}^{2} \mathrm{~d} \Omega^{2}+\int_{\Gamma_{2}^{2}} \mathrm{~g}^{2}\left(\mathrm{v}^{2} \cdot \eta^{2}\right) \mathrm{d} \Gamma_{2}^{2}
\end{aligned}
$$

Then equality (23).

It now follows from $\sigma_{\eta}[v . \eta]=0$ on $\Gamma_{3}$, [u. $\left.\eta\right] \leq 0$ and $\mu \in \mathrm{M}$, then (24).

This Lemma permit us to obtain the following variation problem.

Problem $\mathbf{P}_{\mathbf{m}}$ : Find $\mathrm{u} \in \mathrm{V}$ and $\lambda \in \mathrm{M}$ such that Eq. 25-26:

$$
\begin{aligned}
& \mathrm{a}(\mathrm{u}, \mathrm{v})+\mathrm{b}(\mathrm{v}, \lambda)=\mathrm{L}(\mathrm{v}), \forall \mathrm{v} \in \mathrm{V} \\
& \mathrm{b}(\mathrm{v}, \mu-\lambda) \leq 0, \forall \mu \in \mathrm{M}
\end{aligned}
$$

Remark 1: According to the precedent lemma, it is easy to remark that $\mathrm{P}_{\mathrm{m}}$ is a mixed formulation of the considered problem. If $\mathrm{u}$ is a solution of problem $\mathrm{P}_{1}$, then $\left(\mathrm{u}, \sigma_{\eta}\right)$, where $\sigma^{\ell}=\mathrm{A}^{\ell} \varepsilon\left(\mathrm{u}^{\ell}\right), \ell=1,2$, is a solution of problem $\mathrm{P}_{\mathrm{m}}$.

Remark 2: Another classical formulation of problem $\mathrm{P}_{1}$ is a given by, (Hild and Laborde, 2002), Find u such that Eq. 27:

$$
\mathrm{u} \in \mathrm{U}_{\mathrm{ad}}, \quad \mathrm{a}(\mathrm{u}, \mathrm{v}-\mathrm{u}) \geq \mathrm{L}(\mathrm{v}-\mathrm{u}), \forall \mathrm{v} \in \mathrm{U}_{\mathrm{ad}}
$$

It is easy to verify that the problem (27) has a unique solution, via the Stampachia theorem.
Existence and uniqueness:

Theorem 1: Under the hypotheses (6)-(8), there exists a unique solution $\mathrm{u} \in \mathrm{V}$ of $\mathrm{P}_{1}$.

Proof of theorem 1: Let $\omega=\left(\omega^{1}, \omega^{2}\right) \in \mathrm{V}$, it is easy to prove that the application:

$$
\mathrm{a}(\omega, \mathrm{v})=\left\langle\mathrm{A}^{1}\left(\varepsilon\left(\omega^{1}\right)\right), \varepsilon\left(\mathrm{v}^{1}\right)\right\rangle_{\mathcal{H}^{1}}+\left\langle\mathrm{A}^{2}\left(\varepsilon\left(\omega^{2}\right)\right), \varepsilon\left(\mathrm{v}^{2}\right)\right\rangle_{\mathcal{H}^{2}}
$$

Is a continuous linear form on $\mathrm{V}$ (for $\omega$ fixe), consequently Riesz-Frechet theorem permit us to define the operator $\mathrm{A}: \mathrm{V} \rightarrow \mathrm{V}$, such that:

$$
\langle\mathrm{A} \omega, \mathrm{v}\rangle=\sum_{\ell=1}^{2}\left\langle\mathrm{~A}^{\ell}\left(\varepsilon\left(\omega^{\ell}\right)\right), \varepsilon\left(\mathrm{v}^{\ell}\right)\right\rangle_{\mathcal{H}^{\ell}}, \quad \forall \omega, \mathrm{v} \in \mathrm{V}
$$

Using (6) and Korn inequality, we deduce that the operator A is a strictly monotone and Lipschitz on V. Also $\mathrm{U}_{\mathrm{ad}}$ is a closed, convex and nonempty subset of $\mathrm{V}$.

According to the Lions-Stampachia theorem, we obtain the existence and uniqueness of the element $u \in V$ such that Eq. 20-29:

$$
\mathrm{u} \in \mathrm{U}_{\mathrm{ad}}, \quad\langle\mathrm{Au}, \mathrm{v}-\mathrm{u}\rangle \geq\langle\varphi, \mathrm{v}-\mathrm{u}\rangle, \forall \mathrm{v} \in \mathrm{U}_{\mathrm{ad}}
$$

Then:

$$
\begin{aligned}
& \mathrm{u} \in \mathrm{U}_{\mathrm{ad}}, \sum_{\ell=1}^{2}\left\langle\mathrm{~A}^{\ell}\left(\varepsilon\left(\mathrm{u}^{\ell}\right)\right), \varepsilon\left(\mathrm{v}^{\ell}\right)-\varepsilon\left(\mathrm{u}^{\ell}\right)\right\rangle_{\mathcal{H}^{\ell}} \\
& \geq\langle\varphi, \mathrm{v}-\mathrm{u}\rangle, \forall \mathrm{v} \in \mathrm{U}_{\mathrm{ad}}
\end{aligned}
$$

Theorem 2: Under the hypotheses (6)-(8), there exists a unique solution $\sigma \in \mathcal{H}_{1}$ of $\mathrm{P}_{2}$.

The proof of Theorem 1 and Theorem 2 are carried out in several steps, based on Lions-Stampachia theorem arguments similar to those used in (Lions and Magenes, 1968).

Lemma 3: Let $u$ be the solution of the problem $P_{1}$ obtained in theorem 1 and let $\sigma$ be the solution of the problem $\mathrm{P}_{2}$ obtained in Theorem 2, then $\sigma^{\ell}=\mathrm{F}^{\ell}\left(\varepsilon\left(\mathrm{u}^{\ell}\right)\right)$.

Theorem 3: Under the hypotheses (6)-(8), let $\mathrm{u} \in \mathrm{V}$ and let $\sigma \in \mathcal{H}_{1}$, then we have:

- If $\mathrm{u}$ is a solution of $\mathrm{P}_{1}$ obtained in theorem 1 and $\sigma$ a solution of $\mathrm{P}_{2}$ obtained in theorem 2, then $\sigma^{\ell}=\mathrm{F}^{\ell}\left(\varepsilon\left(\mathrm{u}^{\ell}\right)\right) ; \ell=1,2$ 
- If $\mathrm{u}$ is a solution of $\mathrm{P}_{1}$ obtained in theorem 1 and $\sigma$ $=\left(\sigma^{1}, \sigma^{2}\right)$ with $\sigma^{\ell}=F^{\ell}\left(\varepsilon\left(u^{\ell}\right)\right) ; \ell=1,2$, then $\sigma$ is a solution of $\mathrm{P}_{2}$

- If $\sigma$ is a solution of $\mathrm{P}_{2}$ obtained in theorem2, then there exists a unique solution $\mathrm{u}=\left(\mathrm{u}^{1}, \mathrm{u}^{2}\right) \in \mathrm{V}$ of $\mathrm{P}_{1}$ such that $\sigma^{\ell}=\mathrm{F}^{\ell}\left(\varepsilon\left(\mathrm{u}^{\ell}\right)\right) ; \ell=1,2$

Proof of theorem 3: The proof of Theorem 3, is a result of the lemma 2, theorem 1, theorem 2 and lemma 3.

Theorem 4: Under the hypotheses (6)-(8), there exists a unique solution $(\mathrm{u}, \lambda) \in \mathrm{V} \times \mathrm{M}$ of $\mathrm{P}_{\mathrm{m}}$.

Proof of theorem 4: Let $u \in V$ be the solution of $P_{1}$ obtained in Theoremland led $\sigma=\left(\sigma^{1}, \sigma^{2}\right)$ be the functions given by $\sigma^{\ell}=\mathrm{F}^{\ell}\left(\varepsilon\left(\mathrm{u}^{\ell}\right)\right) ; \ell=1,2$, according to lemma 2 , we have $\sigma_{\eta}^{1}=\sigma_{\eta}^{2} \in M$ and $\left(u, \sigma_{\eta}\right)$ is a solution of $\mathrm{P}_{\mathrm{m}}$. The uniqueness is easly obtained. Let $\left(\mathrm{u}_{1}, \lambda_{1}\right)$ and $\left(\mathrm{u}_{2}, \lambda_{2}\right)$ denote two solutions of $\mathrm{P}_{\mathrm{m}}$.

Then:

$$
\mathrm{a}\left(\mathrm{u}_{1}, \mathrm{v}\right)+\mathrm{b}\left(\mathrm{v}, \lambda_{1}\right)=\mathrm{L}(\mathrm{v})=\mathrm{a}\left(\mathrm{u}_{2}, \mathrm{v}\right)+\mathrm{b}\left(\mathrm{v}, \lambda_{2}\right), \forall \mathrm{v} \in \mathrm{V}
$$

By subtracting, we have Eq. 28:

$$
\mathrm{a}\left(\mathrm{u}_{1}-\mathrm{u}_{2}, \mathrm{v}\right)+\mathrm{b}\left(\mathrm{v}, \lambda_{1}-\lambda_{2}\right)=0
$$

Putting $v=u_{1}-u_{2} \in V$ in (27), we get Eq. 29:

$$
\mathrm{a}\left(\mathrm{u}_{1}-\mathrm{u}_{2}, \mathrm{u}_{1}-\mathrm{u}_{2}\right)+\mathrm{b}\left(\mathrm{u}_{1}-\mathrm{u}_{2}, \lambda_{1}-\lambda_{2}\right)=0
$$

and since a(.,.) is positive, using (26) we conclude:

$$
\begin{aligned}
& 0 \leq \mathrm{a}\left(\mathrm{u}_{1}-\mathrm{u}_{2}, \mathrm{u}_{1}-\mathrm{u}_{2}\right)=-\mathrm{b}\left(\mathrm{u}_{1}-\mathrm{u}_{2}, \lambda_{1}-\lambda_{2}\right)= \\
& \left(\mathrm{b}\left(\mathrm{u}_{1}, \lambda_{2}-\lambda_{1}\right)+\mathrm{b}\left(\mathrm{u}_{2}, \lambda_{1}-\lambda_{2}\right)\right) \leq 0
\end{aligned}
$$

Consequently:

$$
\mathrm{a}\left(\mathrm{u}_{1}-\mathrm{u}_{2}, \mathrm{u}_{1}-\mathrm{u}_{2}\right)=\mathrm{b}\left(\mathrm{u}_{1}-\mathrm{u}_{2}, \lambda_{1}-\lambda_{2}\right)=0
$$

Which implies that $\mathrm{u}_{1}=\mathrm{u}_{2}$ and from (28), we have:

$$
\mathrm{b}\left(\mathrm{v}, \lambda_{1}-\lambda_{2}\right)=0: \forall \mathrm{v} \in \mathrm{V} .
$$

From where, it results $\lambda_{1}=\lambda_{2}$.

Theorem 5: For $\sigma^{\ell}=\mathrm{A}^{\ell} \varepsilon\left(\mathrm{u}^{\ell}\right) ; \ell=1,2$, let $\mathrm{u} \in \mathrm{V}$ and $\lambda \in \mathrm{M}$, the following hypotheses are equivalent:
- $\quad \mathrm{u}$ is a solution of $\mathrm{P}_{1}$ with $\lambda=-\sigma_{\eta}$

- $(\mathrm{u}, \lambda)$ is a solution of $\mathrm{P}_{\mathrm{m}}$

Proof of theorem 5: The implication (i) $\Rightarrow$ (ii) is obvious using the Lemma 2.

Concerning the inverse implication (ii) $\Rightarrow$ (i). Let $\tilde{\mathrm{u}}=\left(\tilde{\mathrm{u}}_{1}, \tilde{\mathrm{u}}_{2}\right)$ denotes the solution of $\mathrm{P}_{1}$ obtained in Theorem 1 and let $\tilde{\sigma}=\left(\tilde{\sigma}_{1}, \tilde{\sigma}_{2}\right)$ be the function given by:

$$
\tilde{\sigma}^{\ell}=\mathrm{A}^{\ell} \varepsilon\left(\tilde{\mathrm{u}}^{\ell}\right), \quad \ell=1,2
$$

According to Lemma 2 we have $\left(\tilde{\mathrm{u}},-\tilde{\sigma}_{\eta}\right)$ is a solution of $\mathrm{P}_{\mathrm{m}}$, which parmit us to conclude that $\mathrm{u}=\tilde{\mathrm{u}}$. From the fact that $\mathrm{P}_{\mathrm{m}}$ has a unique solution, we conclude that $u$ is a solution of $P_{1}$, with $\lambda=-\sigma_{\eta}$.

\section{REFERENCES}

Amassad, A. and M. Sofonea, 1998. Analysis of a quasistatic viscoplastic problem involving tresca friction law. Discrete Contin. Dynam. Syst., 4: 5572.

Djabi, S., M. Sofonea and B. Teniou, 1998. Analysis of some frictionless contact problems for elastic bodies. Ann. Polonici Math.

Drabla, S, 1999. Variational analysis of some boundary value problems in elasticity and viscoplasticity. PhD Thesis, University of Setif.

Hild, P. and P. Labord, 2002. Quadratic finite element methods for unilateral contact problems. Applied Num. Math., 41: 401-421. DOI: 10.1016/S01689274(01)00124-6

Lions, J.L. and E. Magenes, 1968. Problèmes aux Limites non Homogènes et Applications. 1st Edn., Dunod, Paris, pp: 957. 